\title{
Editorial: Vibrionaceae Diversity, Multidrug Resistance and Management
}

\author{
Learn-Han Lee ${ }^{1,2 *}$ and Pendru Raghunath ${ }^{3 *}$ \\ ${ }^{1}$ Novel Bacteria and Drug Discovery Research Group, School of Pharmacy, Monash University Malaysia, Bandar Sunway, \\ Malaysia, ${ }^{2}$ Center of Health Outcomes Research and Therapeutic Safety, School of Pharmaceutical Sciences, University of \\ Phayao, Phayao, Thailand, ${ }^{3}$ Department of Microbiology, School of Medicine, Texila American University, Georgetown, \\ Guyana
}

Keywords: Vibrio sp., multidrug resistance, pathogenesis, virulence, genome, prevalence

\section{Editorial on the Research Topic Vibrionaceae Diversity, Multidrug Resistance and Management}

The genus Vibrio from Vibrionaceae family were originally isolated by an Italian physician, Filippo Pacini in 1854 (Thompson et al., 2004). Presently, the genus contains a total of 142 species which are marine originated and its taxonomy is continuously being revised due to the revelation of new species (Summer et al., 2001; Igbinosa and Okoh, 2008; Sawabe et al., 2013). Some of the Vibrio species are identified as foodborne pathogens causing illnesses upon consumption of contaminated seafood or water (Letchumanan et al., 2014; Law et al., 2015). The three Vibrio species primary for foodborne diseases are Vibrio cholera-associated from food contamination or transmission of infection from person to person, Vibrio parahemolyticus - associated with food contamination, and Vibrio vulnificus-associated with wound infection (Letchumanan et al.). Significant efforts have been applied to analyze the genetic as well as the molecular mechanisms underlying the infection actions of Vibrio.

This Research Topic brings together 19 articles that features the current discoveries concerning the biology of pathogenic Vibrio species covering from the epidemiology studies to discovery of antibiotic resistant strains in the environment which causes immense impact on the treatment and management of illnesses. The genome and non-antibiotic management articles in this research topic provides a deeper knowledge on Vibrio.

As mentioned in four of the articles, Vibrio sp. has a well diverse epidemiology. In Japan, this ecological pathogen can be found among seawaters of coral reefs in Ishigaki. The discoveries uncovered that Ishigaki coral reefs consisted of 22 known and 12 potential novel Vibrionaceae species. The abundance of Vibrio sp. had a significant positive correction with the rising seawater temperature. Amin et al. presented the results that would benefit in the coral conservation and future actions. Where else, in the neighboring nation Shanghai, China, Vibrio parahaemolyticus has been detected as a major pathogen for foodborne gastroenteritis cases. All the V. parahaemolyticus isolated from patients in Shanghai hospital exhibited T3SS1 and T3SS2 genes, 7/42 positive for trh gene and 37/42 positive for $t d h$ gene. Further it was revealed that these clinical isolates expressed multidrug antibiotic resistance and carried resistance genes (Li et al.). Pathogenic Vibrios affecting bivalves in hatcheries has been reviewed by Dubert et al. This review article provided an insight on the Vibrio sp. affecting the hatcheries and proposed effective measures to curb the pathogens. These Vibrios can be managed through proper water treatments method in hatcheries, application of antibiotics, probiosis, quorum quenching, and phage therapy (Dubert et al.). The rarely unexplored $V$. vulnificus occurrence, healthcare burden and prevention were reviewed by Heng et al. $V$. vulnificus has been reported to be infecting in many countries worldwide and caused a huge healthcare burden. Efforts to treat $V$. vulnificus infections has hampered due to the emergence of multidrug resistant strains in the environment. It was suggested that appropriate choice of 
treatment would be cefotaxime and ceftriaxone since the first line of drugs may longer be applicable to treat $V$. vulnificus infections (Heng et al.).

A remarkable feature of $V$. parahaemolyticus's ability of sensing human bile and regulate virulence reviewed by Letchumanan et al. The role of $V$. parahaemolyticus virulence starts upon ingestion of the bacterium and exposures to bile salts. Two encoded protein, VtrA and VtrC will cooperate and form a protein complex on the surface of the membrane of the bacterial cell. This complex structure then binds to bile salts and triggers the cell to produce toxins. Upon binding of bile salts to the VtrA/VtrC complex, the cytoplasmic DNA binding domain of VtrA is activated which in turn induces VtrB to activate, resulting in the T3SS2 expression. T3SS2 virulence is secreted thus causing illness to human. The information on bile-bacteria interaction pathway is important in order to develop drugs that can suppress bacterial virulence (Letchumanan et al.).

The emergence of multidrug resistant strains has become an international health crisis as causing significant threat to human well-being (WHO, 2014; Letchumanan et al., 2015). In Bangladesh, it was reported that $V$. cholerae $\mathrm{O} 1$ was resistant toward ciprofloxacin and azithromycin. These two antibiotics are currently used for prophylactic treatment of $V$. cholerae infections. The reduced susceptibility to ciprofloxacin and azithromycin is alarming for cholera-endemic countries of Asia and Africa (Rashed et al.). In Malaysia, V. parahaemolyticus strains isolated from shellfish was reported to be resistant against second and third lines of antibiotics. Majority of the strains were resistant toward 5 antibiotics out 14 antibiotics tested. These results are a real concern and warrants a continuous surveillance on the antibiotics used in the aquaculture settings (Letchumanan et al.). Similar antibiotic resistant pattern was discovered among $V$. cholerae non-O1/non-O139 collected from Haiti waters. The highest proportion of isolates were reported to be resistant against sulfonamide, amoxicillin, and trimethoprimsulfamethoxazole. There was isolates exhibited resistance toward erythromycin and doxycycline, the two antibiotics used to treat cholerae in Haiti. The presences of $V$. cholerae non-O1/non-O139 with resistance pattern against clinically used antibiotics may cause public health threat (Baron et al.). Pathogenic V. cholerae and $V$. vulnificus with antimicrobial resistance patterns has been reported in Germany. The study further revealed that resistant toward carbapenems was observed in four environmental $V$. cholerae strains studied. Carbapenems are the last line of antibiotics for the treatment of Vibrio infections. The occurrence of carbapenemase producing $V$. cholerae in German coastal waters is of concern and highlights the need for monitoring the use of antibiotics (Bier et al.). In Senegal, antibiotic resistant $V$. cholerae $\mathrm{O} 1 \mathrm{w}$ was isolated during a cholera outbreak. The isolates showed resistance pattern at least four antibiotics tested including trimethoprim-sulfamethoxazole, streptomycin, trimethoprim, and sulfamethoxazole (Sambe-Ba et al.).

Likewise, the genomics of Vibrio navarrensis has been explored via a phylogenetic relationship by performing a single nucleotide polymorphism (SNP) analysis. Such analyses provide an in-depth overview of unique molecular makers and virulence associated genes of Vibrio navarrensis (Schwartz et al.). Wang et al. has applied and study the use of multiple cross displacement implication combined with gold nanoparticlebased lateral flow biosensor for detection of $V$. parahaemolyticus. Based on the findings, the method was reported to be suitable for rapid screening of $V$. parahaemolyticus in clinical, food, and environmental samples (Wang et al.).

The management of Vibrio spp. using non-antibiotic approach has been well addressed in two review articles in this research topic. Streptomyces are soil living bacteria that has been widely recognized as industrially important microorganism due to its potential in producing various antagonistic compounds (e.g., anti-biofilm, anti-quorum sensing, and anti-virulence) against Vibrio pathogens. Streptomyces can be used as probiotic and incorporated in feeds in aquaculture to protect the livestock from pathogens. Despite the fact that there are promising outcomes introduced by past researchers on the use of Streptomyces as a probiotic in aquaculture, further extensive trials are needed to establish the probiotic nature of Streptomyces in disease prevention and development improvement of aquaculture animals (Tan et al.). Essentially, another review article discussed about the advantages of bacteriophages as a nonantibiotic agent. Bacteriophages as a non-antibiotic approach has gained popularity recently due to the increase emergence of antibiotic resistant bacteria worldwide. The bacteriophages have various advantages such as being host specific, environmental friendly, can be effortlessly found and isolated from ecological sources, and cost effective compared to antibiotics. These phages have been proven to control and inhibit virulence of Vibrio sp. isolated from both clinical and environmental. The recognition and safety accreditation by US Food and Drug Administration (FDA) on phages has open the doors for phages to be commercially manufactured for human application and consumption (Letchumanan et al.).

In summary, the collection of manuscripts provided in this Research Topic offers a comprehensive view on the prevalence and antibiotic resistance profiles of Vibrio sp. and as well as discussed, the management methods. This issue shows the significant progress and understanding in the control of pathogenic Vibrio sp. and provides insights to researchers worldwide about new and promising therapies.

\section{AUTHOR CONTRIBUTIONS}

L-HL performed the literature search and writing of the manuscript. PR provided critical review and insight to improve the writing. The research project was conceptualized by L-HL.

\section{FUNDING}

This work was financially supported by MOSTI ScienceFund Grant (Project No. 06-02-10-SF0300) and External Industry Grants from Biotek Abadi Sdn Bhd (vote no. GBA-808138 and GBA-808813) awarded to L-HL.

\section{ACKNOWLEDGMENTS}

Authors acknowledge the sincere effort from Mr. Vengadesh Letchumanan in assisting the completion of this project. 


\section{REFERENCES}

Igbinosa, E. O., and Okoh, A. I. (2008). Emerging Vibrio species: an unending threat to public health in developing countries. Res. Microbiol. 159, 495-506. doi: 10.1016/j.resmic.2008.07.001

Law, J. W.-F., Ab Mutalib, N.-S., Chan, K.-G., and Lee, L.-H. (2015). Rapid methods for the detection of foodborne bacterial pathogens: principles, applications, advantages and limitations. Front. Microbiol. 5:770. doi: $10.3389 /$ fmicb.2014.00770

Letchumanan, V., Chan, K.-G., and Lee, L.-H. (2014). Vibrio parahaemolyticus: a review on the pathogenesis, prevalence, and advance molecular identification techniques. Front. Microbiol. 5:705. doi: 10.3389/fmicb.2014. 00705

Letchumanan, V., Chan, K.-G., and Lee, L.-H. (2015). An insight of traditional plasmid curing in Vibrio species. Front. Microbiol. 6:735. doi: $10.3389 /$ fmicb.2015.00735

Sawabe, T., Ogura, Y., Matsumura, Y., Feng, G., Amin, A. K. M. R., Mino, S., et al. (2013). Updating the Vibrio clades defined by multilocus sequence phylogeny: proposal of eight new clades, and the description of Vibrio tritonius sp. nov. Front. Microbiol. 4:414. doi: 10.3389/fmicb.2013.00414
Summer, J., De Paola, A., Osaka, K., Karunasager, I., Walderhaug, M., and Bowers, J. (2001). "Hazard identification, exposure assessment and hazard characterization of Vibrio spp. in seafood," in Joint FAO/WHO Activities on Risk Assessment of Microbiological Hazards in Foods, ed WHO (Rome: FAO), 1-105. Thompson, F. L., Iida, T., and Swings, J. (2004). Biodiversity of Vibrios. Microbiol. Mol. Biol. Rev. 68, 403-431. doi: 10.1128/MMBR.68.3.403-431.2004

WHO (2014). Antimicrobial Resistance Global Report on Surveillance. World Health Organization. Available online at: http://www.who.int/drugresistance/ documents/surveillancereport/en/

Conflict of Interest Statement: The authors declare that the research was conducted in the absence of any commercial or financial relationships that could be construed as a potential conflict of interest.

Copyright (c) 2018 Lee and Raghunath. This is an open-access article distributed under the terms of the Creative Commons Attribution License (CC BY). The use, distribution or reproduction in other forums is permitted, provided the original author(s) and the copyright owner are credited and that the original publication in this journal is cited, in accordance with accepted academic practice. No use, distribution or reproduction is permitted which does not comply with these terms. 